\title{
Correction to: Lack of adverse effects in subchronic and chronic toxicity/carcinogenicity studies on the glyphosate-resistant genetically modified maize NK603 in Wistar Han RCC rats
}

\author{
Pablo Steinberg ${ }^{1,17} \cdot$ Hilko van der Voet $^{2} \cdot$ Paul W. Goedhart $^{2} \cdot$ Gijs Kleter $^{3} \cdot$ Esther J. Kok $^{3} \cdot$ Maria Pla $^{4,5} \cdot$ Anna Nadal $^{4}$.

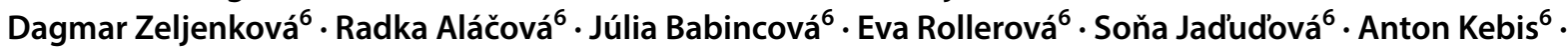 \\ Elena Szabova ${ }^{6}$. Jana Tulinská7 · Aurélia Líšková7 $\cdot$ Melinda Takácsová7 · Miroslava Lehotská Mikušová7 . \\ Zora Krivošíková $^{7} \cdot$ Armin Spök $^{8,9} \cdot$ Monica Racovita $^{9,18}$. Huib de Vriend ${ }^{10} \cdot$ Roger Alison $^{11}$. Clare Alison ${ }^{11}$. \\ Wolfgang Baumgärtner ${ }^{12} \cdot$ Kathrin Becker $^{12}$. Charlotte Lempp ${ }^{12} \cdot$ Marion Schmicke $^{13}$. Dieter Schrenk ${ }^{14}$. \\ Annette Pöting ${ }^{15} \cdot$ Joachim Schiemann ${ }^{16} \cdot$ Ralf Wilhelm $^{16}$
}

Published online: 24 April 2020

(c) Springer-Verlag GmbH Germany, part of Springer Nature 2020

\section{Correction to: Archives of Toxicology (2019) 93:1095-1139 https://doi.org/10.1007/s00204-019-02400-1}

(1) In the original publication, the starting point in time for the three feeding trials was incorrectly given. The first sentence in the second paragraph of the "Rat feeding trials" subchapter in the Materials and methods section (page 1098) should read:

The original article can be found online at https://doi.org/10.1007/ s00204-019-02400-1.

Pablo Steinberg

pablo.steinberg@mri.bund.de

1 Institute for Food Toxicology, University of Veterinary Medicine Hannover, Bischofsholer Damm 15, 30173 Hannover, Germany

2 Wageningen University and Research, Biometris, Droevendaalsesteeg 1, 6708 PB Wageningen, The Netherlands

3 RIKILT Wageningen University \& Research, Akkermaalsbos 2, 6708 WB Wageningen, The Netherlands

4 Institute of Food and Agricultural Technology, University of Girona, Campus Montilivi, 17003 Girona, Spain

5 CRAG-CSIC-IRTA-UAB-UB, Edifici CRAG, Campus UAB, 08193 Cerdanyola, Spain

6 Slovak Medical University, Faculty of Public Health, Limbová 12, 83303 Bratislava, Slovakia

7 Slovak Medical University, Faculty of Medicine, Limbová 12, 83303 Bratislava, Slovakia

8 Graz University of Technology, Schlögelgasse 2, 8010 Graz, Austria
"The first 90-day feeding trial and the chronic toxicity/ carcinogenicity study were started $2-3$ weeks and the second 90-day feeding trial was started 1-2 weeks after delivery of the animals at the animal testing facility".

(2) In the original publication, Figs. 5 and 6 (page 1118) showed, for all points in time, the mean body weight and the food consumption data, respectively, of only the male and

9 Alpen-Adria Universität Klagenfurt, Schlögelgasse 2, 8010 Graz, Austria

10 LIS Consult, Hogesteeg 9, 3972 JS Driebergen, The Netherlands

11 Roger Alison Ltd., Lampeter, Ceredigion, UK

12 Department of Pathology, University of Veterinary Medicine Hannover, Bünteweg 17, 30559 Hannover, Germany

13 Clinic for Cattle, University of Veterinary Medicine Hannover, Bischofsholer Damm 15, 30173 Hannover, Germany

14 Food Chemistry and Toxicology, University of Kaiserslautern, Erwin-Schrödinger-Straße 52, 67663 Kaiserslautern, Germany

15 Federal Institute for Risk Assessment, Max-Dohrn-Straße 8-10, 10589 Berlin, Germany

16 Institute for Biosafety in Plant Biotechnology, Julius Kühn-Institut (JKI), Federal Research Centre for Cultivated Plants, Erwin-Baur-Str. 27, 06484 Quedlinburg, Germany

17 Present Address: Max Rubner-Institut, Federal Research Institute of Nutrition and Food, Haid-und-Neu-Str. 9, 76131 Karlsruhe, Germany

18 Present Address: Global Sustainability Institute, Anglia Ruskin University, East Road, Cambridge CB1 1PT, UK 
Fig. 5 Mean body weight of all male and female rats surviving until the points in time plotted in the combined chronic toxicity/carcinogenicity feeding trial with GM maize NK603 at an inclusion rate of 11 and $33 \%$ in the diet
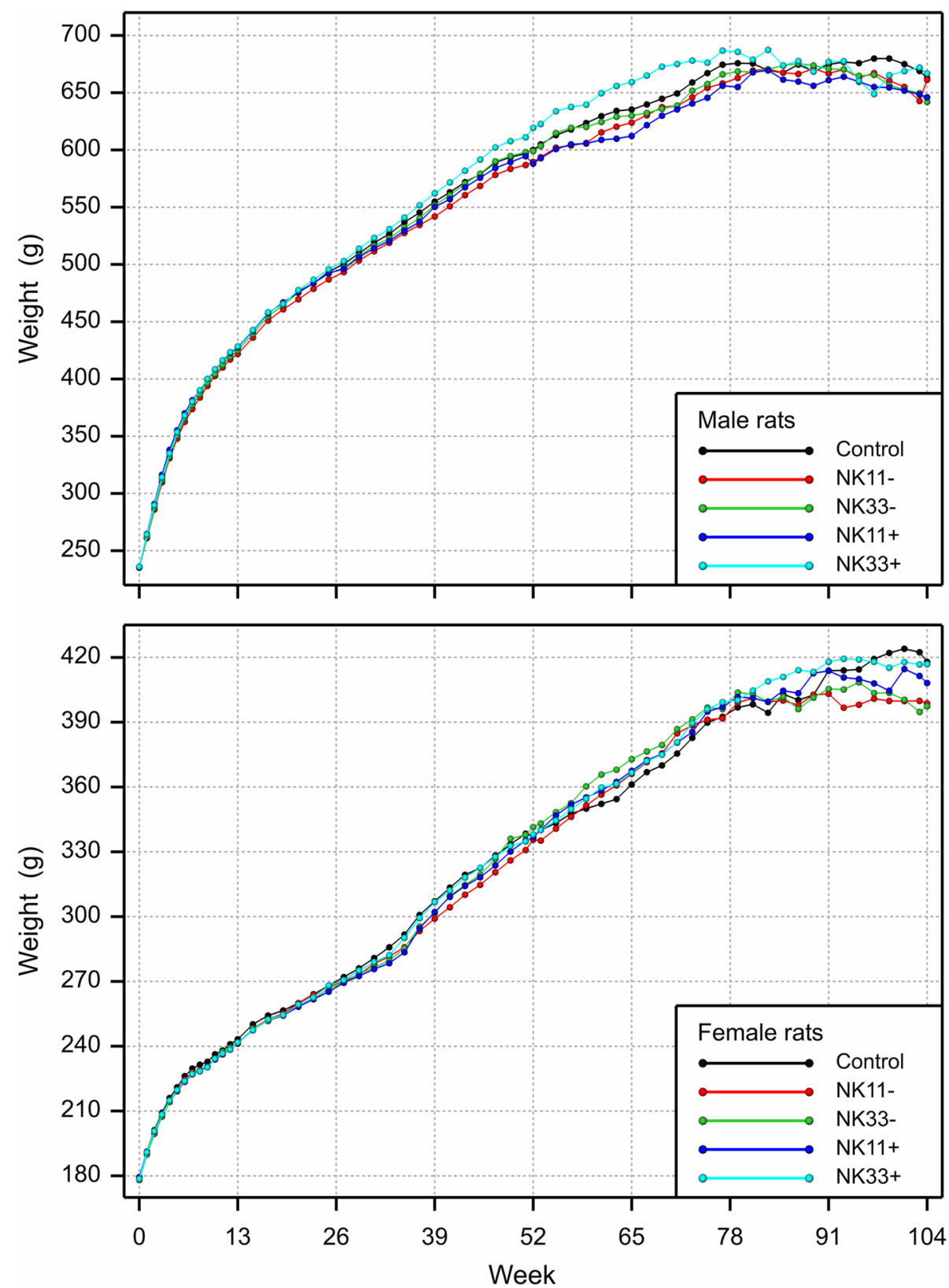

female rats that survived until the end of the chronic toxicity/ carcinogenicity study. The figure legends refer, however, to another option, where all available data are used to calculate mean body weights and feed consumptions, and these legends describe the figures that we intended to publish. The current Figs. 5 and 6 should, therefore, be replaced by the accompanying new Figs. 5 and 6.

Consistent with these new figures, two changes in the text have to be made as follows:

(I) The second and third sentences in the second paragraph of the "Combined chronic toxicity/carcinogenicity feeding trial with the GM maize NK603 at an inclusion rate of 11 and $33 \%$ in the diets" subchapter in the Results section on page 1117 should be substituted by the following sentence:

"In weeks 52-78, the male rats fed the NK603 + Roundup diet at an inclusion rate of $33 \%$ showed a higher mean body weight and feed consumption when compared to the corresponding control group, while the mean body weight and feed consumption of the female rats were similar in all five experimental groups (Figs. 5 and 6)".

(II) In the Discussion section, the third sentence in the second paragraph of page 1134 should be substituted by the following sentence: 
Fig. 6 Mean feed consumption of all male and female rats surviving until the points in time plotted in the combined chronic toxicity/carcinogenicity feeding trial with GM maize NK603 at an inclusion rate of 11 and $33 \%$ in the diet
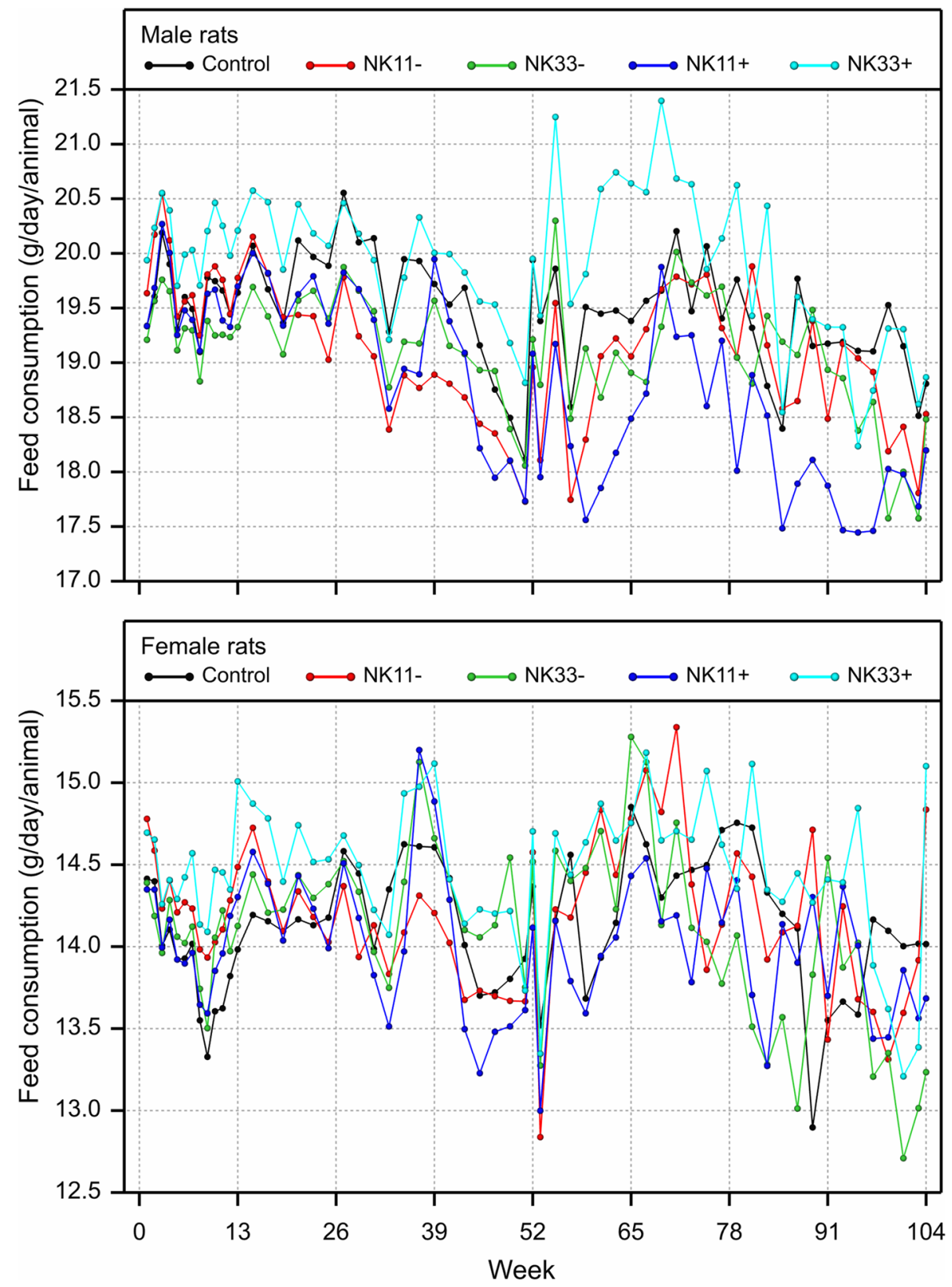

"The increased mortality observed between the 12th and 24 th month of the feeding trial in male rats fed the $33 \%$ NK603 + Roundup diet coincided with an increase in the body weight and feed consumption".

Please note that these revisions do not affect in any way the overall conclusions on the lack of any NK603 maizerelated adverse effects. Finally, we would like to thank Dr. Graham Tobin, formerly the Technical Director for Envigo
Teklad diets and Global QA Director at Harlan, for his extremely helpful comments.

Publisher's Note Springer Nature remains neutral with regard to jurisdictional claims in published maps and institutional affiliations. 